\title{
Familiarity and Complexity during a Takeover in Highly Automated Driving
}

\author{
Marlene Susanne Lisa Scharfe-Scherf ${ }^{1,2}$ (D) . Nele Russwinkel ${ }^{2}$ \\ Received: 16 December 2020 / Revised: 2 April 2021 / Accepted: 17 May 2021 / Published online: 21 July 2021 \\ (C) The Author(s) 2021
}

\begin{abstract}
This paper shows, how objective complexity and familiarity impact the subjective complexity and the time to make an action decision during the takeover task in a highly automated driving scenario. In the next generation of highly automated driving the driver remains as fallback and has to take over the driving task whenever the system reaches a limit. It is thus highly important to develop an assistance system that supports the individual driver based on information about the drivers' current cognitive state. The impact of familiarity and complexity (objective and subjective) on the time to make an action decision during a takeover is investigated. To produce replicable driving scenarios and manipulate the independent variables situation familiarity and objective complexity, a driving simulator is used. Results show that the familiarity with a traffic situation as well as the objective complexity of the environment significantly influence the subjective complexity and the time to make an action decision. Furthermore, it is shown that the subjective complexity is a mediator variable between objective complexity/familiarity and the time to make an action decision. Complexity and familiarity are thus important parameters that have to be considered in the development of highly automated driving systems. Based on the presented mediation effect, the opportunity of gathering the drivers' subjective complexity and adapting cognitive assistance systems accordingly is opened up. The results of this study provide a solid basis that enables an individualization of the takeover by implementing useful cognitive modeling to individualize cognitive assistance systems for highly automated driving.
\end{abstract}

Keywords Highly automated driving · Takeover request $\cdot$ Complexity $\cdot$ Situation awareness $\cdot$ Familiarity

Cognitive assistance

\section{Introduction}

Although highly automated driving is developing fast, in the next two generations of driving automation, the driver will remain as fallback if the automation reaches its limit [15, 23]. Hence, it becomes highly important to keep the human operator informed and able to interact with the system effectively and safely [6]. The importance of generating intelligent assistance systems thus rises.

Marlene Susanne Lisa Scharfe-Scherf

marlene-susanne-lisa.scharfe@ de.bosch.com

1 Robert Bosch GmbH, Robert-Bosch-Allee 1, 74232 Abstatt, Germany

2 Department of Psychology and Ergonomics, TU Berlin, Marchstr. 23, 10587 Berlin, Germany
Recent studies present first approaches towards systems that assist drivers based on the current situation. [29], for example, propose an adaptive system that supports by presenting the approach information of other vehicles through peripheral vision with color information. Furthermore, collisions at intersections can be avoided and the driver's reaction time can be reduced by informing drivers about the existence of oncoming vehicles using right-turn timing assistance [30]. However, an important aspect that needs to be considered when designing driver assistance systems is the real-time assessment of the state of the driver [1]. [26] show that a crossing-assistance system for visually disabled persons at signalized intersections enables the visually disabled to cross safely at signalized intersections under actual road conditions. Such adaptive driving assistance is extremely critical for safe driving environment.

With the next level of automation (Level 3, conditional automation; [23]), the driver is allowed to turn away from the driving task during the automated drive. Still, the driver 
remains as fallback and has to intervene as soon as the vehicle triggers a takeover request. As drivers become passive, cognitive engagement is reduced. [6] identifies this passiveness as one of three main factors that create a loss of situation awareness. Further factors are a poor design of system interfaces and the challenges of lowered vigilance in monitoring automated systems [6]. To take over driving readily with concentration, [12] propose a humanmachine interaction system to induce a smooth and safe transition to manual driving from Level 3 automated driving with using voice guidance, alarm sound and interior lights. However, to really provide safety during the takeover in highly automated driving, cognitive assistance systems have to be adapted to the individual driver. Those assistance systems should be able to incorporate mental calculations, communication, assessment of relevant information and scanning for traffic [8]. By cooperating with the driver and assuring the mutual understanding between human-agents and machine-agents, conflicts can be reduced or avoided [2]. Hence, it becomes crucial to understand the current drivers state of situation awareness.

According to [7], situation awareness is divided into three levels. The first level is the perception of elements in the current situation. In driving, the visual perception is the most important human sense, including elements like receptors, sensory-memory perception, processes of feature integration and cognitive processes [20]. In driving, the perception level thus constitutes a main part regarding the takeover. Based on the perception, the comprehension of the current situation is formed [7]. In this paper, the two levels are understood as the perception of vehicles in the traffic environment and the consideration and selection of vehicles that are relevant for the current situation. Perception and integration of present vehicles can be identified as main reason for a longer process of regaining situation awareness [21]. Hence, especially concerning the underlying cognitive processes these two levels are closely attended in this paper. The projection of the future status is based on perception and comprehension [7]. The three levels overlap and are not strictly separated, but they build up on each other. As soon as the situation awareness representation of a driver is updated, the driver is able to make a decision about necessary actions. The time until a driver makes an action decision marks a crucial point. It represents the point in time when a first situation representation is build up that the driver bases the action decision on. As it is important to provide high controllability over the vehicle and enable drivers to take over safe and comfortable, the driver must be enabled to properly build up a useful situation awareness representation to base the decision on before executing the action and fully taking over all aspects of the vehicle control.

\subsection{Definition of Relevant Constructs}

Several approaches to define situation complexity exist (e.g. [3, 5, 10, 19, 21, 25]). These differ strongly in their concept of situation complexity. Types of situations can be distinguished in various ways based on objective characteristics like the traffic situation, a type of traffic environment or a weather condition ( [24]; objective complexity). Based on [19], the objective complexity can vary with road geometry (rectilinear vs. curvilinear), the roadside environments (quantity and variability of traffic signs, variability of scenery) and traffic density (low vs. high). [5] showed that less complex scenarios result in shorter takeover times and a higher takeover quality. In their study, the complexity of situations differ in their demand of the necessary reaction (e.g. a lane change, avoiding obstacles) but do not indicate other aspects that add to situation complexity. The role that objective characteristics play in driving is thus very important. Especially concerning the update of situation awareness, objective characteristics have to be considered during the takeover in highly automated driving. But similar driving situations that differ in their type of objective complexity can lead to differences in the perception of task complexity and performance. [10] found, that people with a higher expertise perceive the same task less variable and more analyzable, than novices and perform better on the task. Hence, objective characteristics can be perceived differently by individual drivers that are taking over the driving task. Each individual driver has a subjective perception of the situation and its complexity (subjective complexity) that additionally influences the process of building up situation awareness. The subjective complexity is dependent on the observer (here the driver) and thus variable. Due to [25] subjective complexity can be understood as a result of a particular perception of a situation or resulting from a distinction between expectation and situation development. [21] found that the regaining of situation awareness during a takeover depends to a large extent on the subjective complexity of current traffic situation. Participants rated the overall complexity of a takeover situation. The study shows that situations with a higher complexity feature a higher mean takeover time [21]. This is an important finding, but lacks in defining which aspects add up to subjective complexity. In order to differentiate the complexity of decision situations for the takeover in highly automated driving, the current paper divides objective and subjective complexity. However, focusing on situation awareness, objective complexity characteristics like weather condition or road geometry play an indirect role. The aspect traffic density in contrast is highly important when building up situation awareness. Altogether, the representation of the 
takeover request (TOR), the objective traffic situation and the current state of the driver play a main role. Based on the above described differentiations, subjective and objective complexity are defined in the context of highly automated driving in the following.

\subsubsection{Objective Complexity}

Similar to [17], objective complexity of a decision situation is in this paper defined as the amount of relevant objects in a certain situation. These objects are located within a predefined area in relation to the ego vehicle. In addition, the in-vehicle environment can add to the objective complexity, including car multimedia, HMI (human-machine interface) and non-driving related tasks (NDRT). In this study the latter are held constant and are thus not further regarded in terms of objective complexity. All objects can vary in their amount, attention stimulation (e.g. size, color) and relevance in the current situation. Here, the complexity of the traffic environment is reduced to the amount of relevant vehicles. For the investigation of the impact of the traffic environment during a takeover, the amount of relevant vehicles is varied. Hence, the influence of the in-vehicle environment is held constant and not investigated.

\subsubsection{Subjective Complexity}

The subjective complexity of a decision situation in this study is defined as the individual perception of complexity in a certain traffic situation. It is dependent on driving experience and situation complexity [10,19]. This includes the amount of environmental cues (here vehicles in the traffic environment) and of objects that are perceived and selected as relevant to draw the attention to. In addition, the current cognitive state (state of situation awareness, occupation etc.) of the driver influences the subjectively perceived complexity. [10] showed, that the perception of a driving task complexity varies with the amount of expertise a driver has. Here, the impact of the NDRT is also not regarded as it is held constant. Thus, the influence it may have on an individuals perception of subjective complexity is equal over all trials. Here, we do not refer to expertise, but rather the familiarity (automatism vs. conscious thought) with the situation and the current task as relevant factors. The subjective complexity is task- and resource-dependent and describes an individuals' subjective perception of complexity in a certain traffic situation. It is not measurable as easy as the objective complexity. Workload questionnaires, eye movement patterns and physiological measurements pose an option to quantify subjective complexity.

\subsubsection{Familiarity with a Situation}

As mentioned above, the familiarity with a situation plays an important role during the takeover in highly automated driving. A lack of familiarity is assumed to increase the subjective complexity. Experts and novices pay attention to different aspects of a task that affects the perception of task complexity and the performance on the task. Depending on the match between a drivers expertise and the demands of the task, experts may show superior performance than novices [10]. Expertise can be referred to different parts of driving and is understood here as a more general description of a driver. In contrast to familiarity, expertise includes more factors of the whole driving task over all situations. Especially, when addressing different traffic situations during a takeover, it is rather familiarity than expertise that needs to be considered. A driver may be more familiar with a certain situation than others, but may lack this familiarity in other situations. The expertise of a driver can thus in general be high, but regarding certain situations there might still be a lack of familiarity. Due to this, the term familiarity rather than expertise is used here. The perception of a situations' complexity is thus dependent on the level of familiarity a driver has with a certain situation. In this study, scenarios vary in their objective complexity (amount of relevant vehicles in the surrounding traffic environment) and the most efficient maneuver decision (left, follow, right). The drivers can thus get familiar with certain vehicle constellations and react more confidently in certain situations. It has been shown by [14] that the overall response time increases when driving with support systems. However, this increase is significantly lower for drivers who are familiar with the system [14]. Dealing with human-machine interaction in driving, the effect of familiarity becomes important. It is relevant to understand in what way the familiarity influences different cognitive mechanisms during the takeover. In this paper, the effect of familiarity with certain driving situations is investigated. It is to understand the impact that familiarity has on the takeover. This includes perceptions of complexity, the time to build a situation representation and to make an action decision (Fig. 1).

\subsection{Research Questions and Hypotheses}

A deep understanding of the underlying cognitive processes, environmental and personal factors (here objective complexity and situation familiarity) and the individual perception of these factors (subjective complexity) are needed to build useful models for the takeover procedure. Based on such models, cognitive states can be predicted and cognitive assistance systems developed. These provide support 
Fig. 1 Hypotheses (H) that are investigated in this study. Dotted lines indicate mediation effects (Source: own figure)

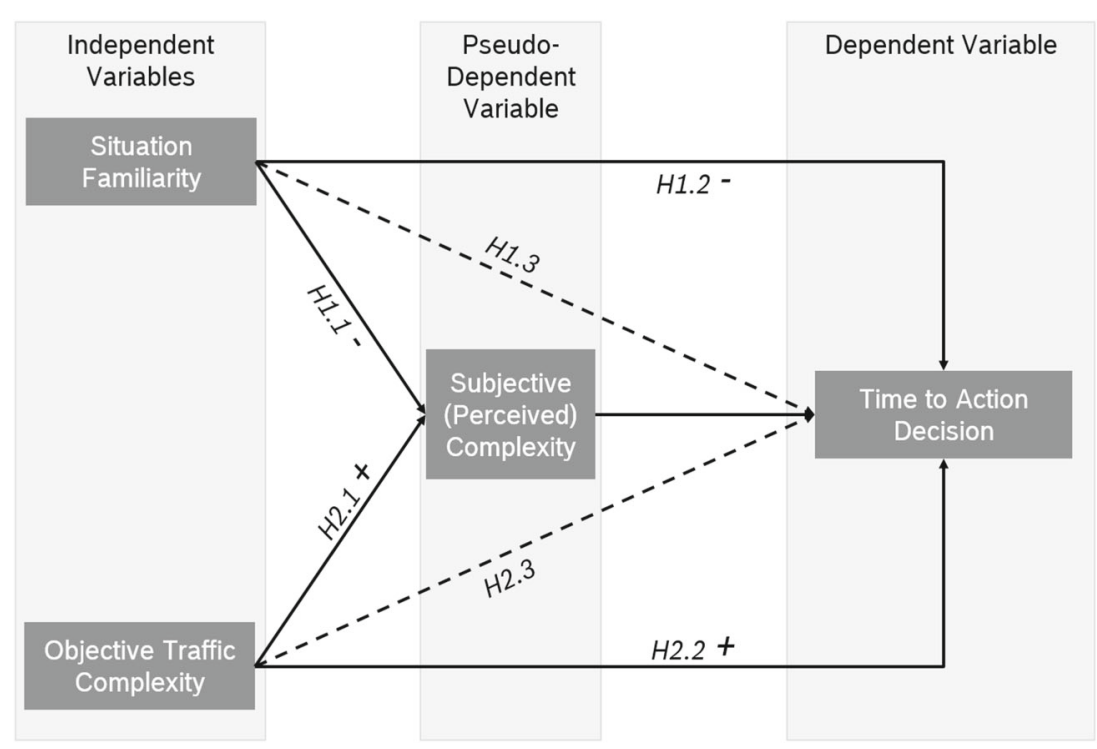

to the driver during a takeover in highly automated driving. Concerning the HMI, the degree of handing over the driving task and lateral and longitudinal intervention can be adapted based on cognitive modeling predictions. In order to develop such cognitive assistance systems that predict and support the driver during the takeover, it is very important to understand the impact of familiarity and objective complexity. In this study, their impact on the subjective complexity and the time to make an action decision when taking over is investigated (Fig. 1). The point in time when an action decision is made is assumed to indicate that a situation model exists, hence the situation awareness is updated. Based on the above discussed limits of current research, the following research questions are addressed in this study and hypothesized relationships examined (Fig. 1).

1. How does the familiarity with a traffic situation influence the perceived complexity during the takeover? And what temporal effect does familiarity with a situation have on the action decision?

- H1.1: The perceived complexity of a situation declines with a rise in familiarity due to repetition of the situation.

- H1.2: Higher familiarity with a situation leads to shorter action decision times.

- H1.3: The influence of situation familiarity on the action decision time is mediated by the perceived complexity of a situation.

2. Does a high objective complexity also lead to a high perceived subjective complexity? If so, how does the amount of relevant vehicles in the traffic environment (objective complexity of a traffic situation) influence the perception of a situation (subjective complexity)?
And what effect does it have on the time to make an action decision?

- H2.1: More relevant vehicles in the traffic environment lead to an increased subjective complexity during a takeover.

- H2.2: In a more complex driving environment, drivers need more time to update their situation awareness model and thus make a later action decision.

- H2.3: The influence of the objective complexity on the time to make an action decision is mediated by the subjective complexity of the situation.

\section{Methods}

In the following section, the simulator that is used for the study is described as well as the study design and materials used to investigate the hypothesized effects. The implementation of the study is approved by the ethics committee of the TU Berlin in April 2019 and Robert Bosch $\mathrm{GmbH}$.

\subsection{Simulator}

The driving simulator that is used consists of six monitors, that simulate a 360 degrees view (Fig. 2). For traffic and scenario simulation, the driving simulation SILAB [28] is used. To make it comparable to driving a car on the road, the driving unit is movable and tilts in accordance to the visual simulation. The HMI is equipped with a speedometer and a visual display for notifications. Indicators are located on the steering wheel. Further, the driving unit contains 


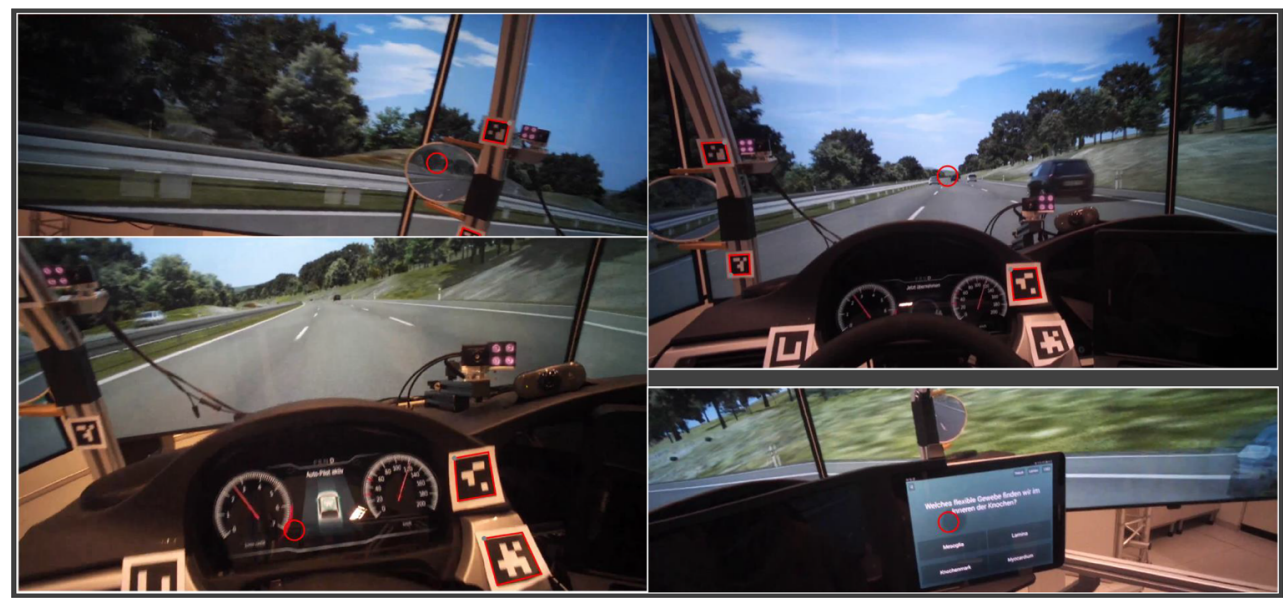

Fig. 2 Exemplary driver view of the driving simulator measured by eye tracking glasses. Representation of left mirror (top left), main view (top right), HMI (bottom left) and mounted tablet with quiz (bottom right). Red circle indicates gaze fixation (Source: own figure)

mirrors, pedals, the handbrake and a fixed mount for tablets. The simulator is equipped with a sound system for driving sounds and speech output.

\subsection{Study Design}

The study is an experimental laboratory study as it is conducted in a driving simulator. This means, that participants are tested in a controlled environment rather than real traffic. The driving simulator has the advantage to enable the testing of several participants under exactly the same traffic conditions. In this case this is highly important as the impact of the surrounding traffic and familiarity effects have to be controlled.

\subsection{Scenarios}

The driving simulation SILAB [28] allows a very precise generation of the six different scenarios that have been tested within this study (Fig. 3). As shown in Fig. 3, actions and their timing in different takeover situations have been tested. The takeover is always triggered when the ego vehicle is on the center lane of the highway with a speed of $120 \mathrm{~km} / \mathrm{h}$. No reason for the takeover is given in this study to prevent unwanted side effects and it is not relevant for what reason the takeover request is triggered. However, the takeover request is always an uncritical one (e.g. exit highway, construction zone ahead). The surrounding traffic is set up for three maneuver options, each with a relatively high or low complexity, resulting in overall six scenarios (Fig. 3). To trigger the corresponding maneuvers, the surrounding traffic is set up accordingly. In order to prevent participants from preparing the maneuver, the traffic constellation is changed as soon as the takeover request appears. Thus, even if participants do not attend the NDRT completely, they cannot prepare the upcoming scenario in advance. Scenarios are set up in the following way. The amount of relevant vehicles per scenario is referred to as RV (relevant vehicles) in the following. Vehicles are

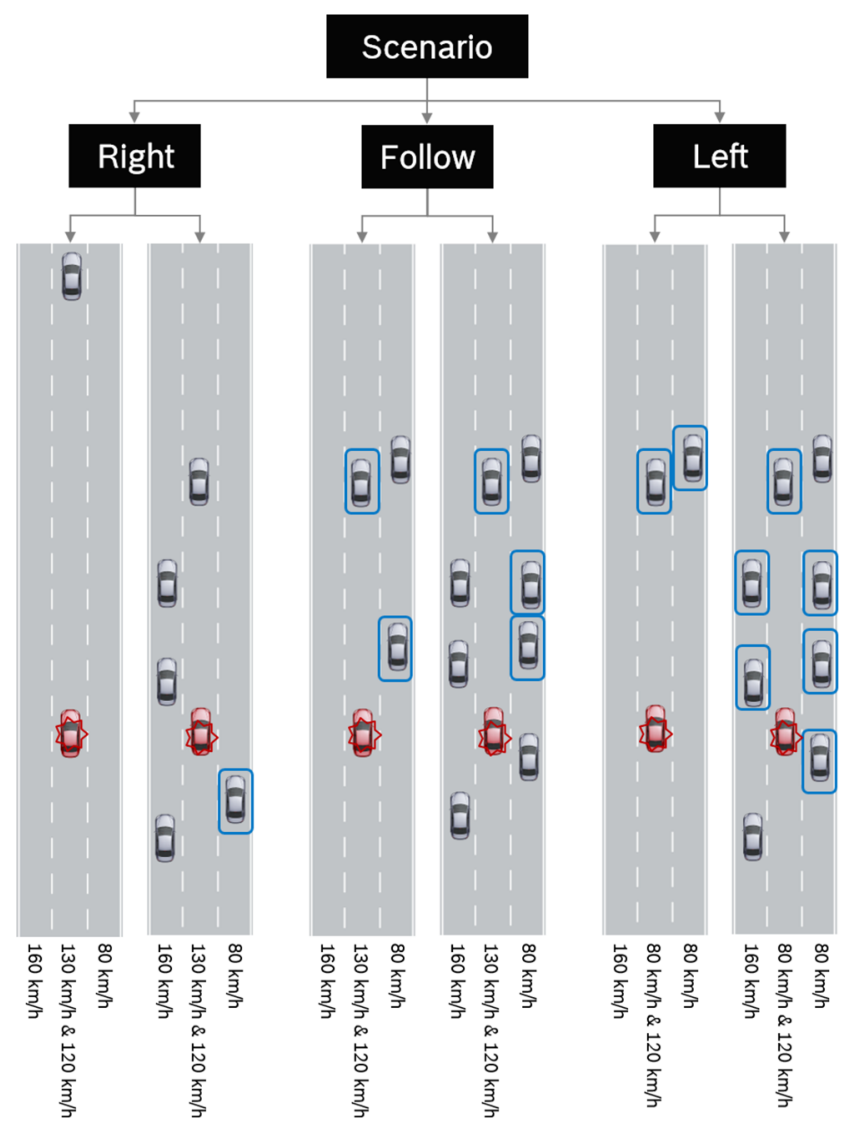

Fig. 3 Traffic scenarios during the takeover request when participants have to take over the driving task. Blue squares mark relevant vehicles in the given scenario situation, the red star marks the ego vehicle (Source: own figure) 
rated as relevant when they are directly relevant for the corresponding maneuver and have to be perceived for a safe maneuver performance. The maneuvers include a lane change to the right (RIGHT), car following (FOLLOW) and a lane change to the left (LEFT). The lowest complexity have the RIGHT scenarios. Cases, where a lane change to the right is necessary are set up with no RV on the oncoming right lane (obligation to drive on the right). In one scenario no relevant traffic surrounds the ego vehicle $(0 \mathrm{RV})$, in the other scenario a slower car in the right back $(80 \mathrm{~km} / \mathrm{h})$ is relevant $(1 \mathrm{RV})$. In the FOLLOW scenarios the right lane is always occupied and the vehicle in the front drives faster $(130 \mathrm{~km} / \mathrm{h})$ than the ego vehicle. In the one of the FOLLOW scenarios only the relevant vehicle on the right lane $(80 \mathrm{~km} / \mathrm{h})$ and one in the front are set up $(130 \mathrm{~km} / \mathrm{h}$; $2 \mathrm{RV})$. In the other FOLLOW scenario, the left lane is occupied as well $(160 \mathrm{~km} / \mathrm{h} ; 3 \mathrm{RV})$. In the LEFT scenarios, the right lane is occupied $(80 \mathrm{~km} / \mathrm{h})$ and the vehicle in the front is obviously slower $(80 \mathrm{~km} / \mathrm{h})$ than the ego vehicle. In the one scenario, only the two relevant vehicles in the front and on the right lane are set up (2RV). In the other scenario, the left and right lane are highly occupied (6RV; Fig. 3). Overall three blocks are executed, in which each scenario appears once. The sequence of the scenarios in each block is randomized, resulting in three different blocks. Additionally, each participant is set up at a different point of the first block to have randomized trials and prevent learning effects of an order.

\subsection{Variables and Measurement}

Four variables are focused on in the study. The two independent variables are the familiarity with the situation (situation familiarity) and the objective complexity of the traffic environment (amount of relevant vehicles; objective traffic complexity). The subjective complexity is a pseudo-dependent variable, as shown in (Fig. 1). Hypothesized is that the subjective complexity is influenced by the two independent variables that are manipulated. In addition, subjective complexity is assumed to have an impact on the dependent variable, the time to action decision. Hence, it is also assumed to be a mediator variable. Variables and measurement methods are further described in detail.

\subsubsection{Situation Familiarity}

The familiarity with a situation is implemented by a repetition of the scenarios. Participants drive through all of the six scenarios three times. Thus they have the lowest familiarity is in the first repetition of each scenario, and the highest familiarity in the third repetition of each one. As in real traffic a situation is never the same, situation familiarity is chosen as term instead of learning. In contrast to learning, no representation of the same facts can be learned for traffic situations. The habituation to general traffic situations is called situation familiarity and rises with repeated exposure. To exclude learning effects of the takeover and HMI representations, a learning session is executed prior to the experiment.

\subsubsection{Objective Traffic Complexity}

The objective complexity of the traffic environment is in this paper based on the amount of relevant vehicles in relation to the ego vehicle. In the conditions it is either high or low. In the low complexity cases only vehicles that are relevant to trigger the corresponding maneuver are used. In complex scenarios as much vehicles as possible in the corresponding scenario are set up. Still, only vehicles, that need to be attended to execute the maneuver-based action count as relevant vehicles. Their distribution and relevance are explained in the following. In Section 2.3, the distribution and the set up is described. This section focuses on the relevance of the vehicles that add up to the objective complexity. The amount of relevant vehicles for the RIGHTLOW scenario is zero $(0 \mathrm{RV})$, as there is no vehicle that is relevant for the current maneuver. The RIGHT-HIGH scenario includes one relevant vehicle, located in the right back of the ego vehicle. Thus, the mirror has to be checked and decided whether the lane change can be executed or not under the given condition (1RV). In the FOLLOWLOW scenario two vehicles are relevant for the action decision. These are the vehicle on the right, indicating that the right lane is occupied and the car in front, which has a higher speed than the ego vehicle (2RV), giving no need for overtaking. The same applies for the FOLLOW-HIGH scenario with an additional vehicle on the right lane in the maneuver relevant area (3RV). Only two vehicles are relevant in the LEFT-LOW scenario. That is the vehicle on the right, indicating that no lane change to the right can be executed and the car in front which has a significantly lower speed than the ego vehicle (speed difference of $40 \mathrm{~km} / \mathrm{h}$ ). Hence, the ego vehicle approaches the oncoming vehicle very fast and a lane change to the left has to be executed $(2 \mathrm{RV})$ in order to avoid strong braking. In the LEFT-HIGH scenario this is the same case, except for another close vehicle on the right lane and three vehicles on the left lane: two of them in front of the ego vehicle and one in the back that is overtaking. All three are driving with a speed of $160 \mathrm{~km} / \mathrm{h}$. These vehicles have to be considered for the maneuver execution. With six relevant vehicles the LEFT-HIGH scenario represents the one with the highest objective complexity (6RV; Fig. 3). As different amounts of vehicles are relevant depending on the maneuver, a distinction between the amount of relevant vehicles is done 
rather than differentiating between high and low conditions only. The benefit is that a more precise and interval-scaled distinction can be drawn rather than a nominal one.

\subsubsection{Subjective Complexity}

The subjective complexity indicates how complex the participants perceive the scenario. In order to assess the subjective complexity, the rating sheet of the NASA-TLX (NASA Task Load Index; [11]) is used. It is a multidimensional rating procedure, including six subscales that are rated on a 20-point likert scale. The NASA-TLX was originally developed to measure workload. Although subjective complexity is not the same as workload, the NASA-TLX is useful to measure subjective complexity in this study due to its items. The items that are addressed are presumed to be indicators of subjective complexity. The first item mental demand is indicated by "How much mental and perceptual activity was required (eg. thinking, deciding, calculating, remembering, looking, searching, etc)? Was the task easy or demanding, simple or complex, exacting or forgiving?". Physical demand is described as: "How much physical activity was required (e.g. pushing, pulling, turning, controlling, activating, etc.)? Was the task easy or demanding, slow or brisk, slack or strenuous restful or laborious?". The third item temporal demand is indicated by "How much time pressure did you feel due to the rate or pace at which the tasks or task elements occurred? Was the pace slow and leisurely or rapid and frantic?", performance by "How successful do you think you were in accomplishing the goals of the task set by the experimenter (or yourself)? How satisfied were you with your performance in accomplishing these goals?", effort by "How hard did you have to work (mentally and physically) to accomplish your level of performance?" and frustration by "How insecure, discouraged, irritated, stressed and annoyed versus secure, gratified, content, relaxed and complacent did you feel during the task?" [11]. The weighting of the items has been criticized in the past [9]. Therefor it is not used in this study. Based on the evaluation it would neither be beneficial for the current purpose. Participants answered the NASA-TLX after each trial, resulting in overall 18 ratings for each scale of the NASA-TLX.

\subsubsection{Time to Action Decision}

The subjective complexity is further assumed to have an impact on the resulting time to make an action decision. Participants have to indicate their action decision aloud as soon as it is made. Hence, after every takeover, participants indicate verbally the action decision they make at the same moment. This method is chosen to gather the time of the actual action decision rather then the time of the action execution. This is due to the fact that the action execution is dependent on the surrounding traffic and cannot always be executed directly when the action decision is made. Hence, by using the verbal indication, the time of the decision to execute a maneuver is directly measured. Based on the action decision time, the time to build up a situation model can be deduced.

\subsection{Procedure}

After a short introduction, participants fill out the formalities (declaration of consent, participant code for complete anonymisation and participant information). Before starting the actual data acquisition, participants read the instructions. To get used to the simulator dynamics and to exclude learning effects of the takeover and the simulator functions, participants practice the takeover before starting the experiment. Finally, the questionnaire for sociodemographic data is filled out.

In the experiment, participants start on a parking lot and have to drive onto a three lane highway. Participants are instructed to activate the highway pilot on the middle lane as soon as it is available. During the automated drive, a quiz is available on the mounted tablet at the center console. On this, participants engage into the non-drivingrelated task while the automated mode is activated. As soon as a takeover request (TOR) is triggered (always at a speed of $120 \mathrm{~km} / \mathrm{h}$ ), participants are advised to stop answering the quiz immediately. No further action for quiz deactivation is needed. Participants can just stop playing and turn their attention away from the quiz. Instructions declare to take over the driving task and to try maintain the speed of $120 \mathrm{~km} / \mathrm{h}$. The action participants decide to execute must be verbalized clearly as soon as the decision is made. The action decision is dependent on the surrounding traffic and German driving law (especially the obligation to drive on the right). The speed should be held as constantly as possible, thus using mainly the steering wheel for the maneuver. After the maneuver, participants are instructed to head for the oncoming parking lot where they come to a stop and fill out the NASA-TLX questionnaire to measure subjective complexity of the preceding scenario. Starting from the parking lot again, participants resume driving into the next scenario. This is repeated 18 times, including six scenarios in randomized order per block. This results in three blocks, that participants drive through. Each block has a different randomized order and each participant starts at a different set-up point of the first block. During the experiment, the time of the action decision is measured with a key press. As soon, as the participant indicates the action decision, the investigator presses the space key on the keyboard. 
The study was conducted in April and May 2019 in a simulator of Robert Bosch $\mathrm{GmbH}$ in Renningen. In a prestudy the test design, methods and technical functionality were tested. The final results base on $N=20$ participants that took part in the main study. Out of the 20 participants 13 are male and 7 female with a mean age of 26.2 years $(S D=2.69)$. Most of the participants drive a car daily $(N=9)$. The others have a driving frequency distributed between five to six times a week and less than once a week. The most common average driving duration per drive is 30 minutes, ranging from 15 to 120 minutes. Highways are used mostly $(N=7)$, followed by Rural Roads $(N=$ $4)$ and cities $(N=2)$. Seven participants did not indicate their most common road usage. All participants are regular drivers and provide existing pre-knowledge of highway situations, although varying in the amount of highway usage. Overall 13 participants indicate to drive moderately. Three participants have a defensive driving style and four state to drive mainly sporty.

\subsection{Statistical Evaluation}

To test whether complexity conditions high vs. low differ significantly from each other, the Mann-Whitney-U test [18] is used. This is due to the fact that although visually the data seems to be distributed normally, Shapiro-Wilk normality [22] tests do not support the assumption of normal distribution in all conditions. Regression analysis is based on the regression equation $y=x \beta+\epsilon(\beta=$ slope $; \epsilon=$ error $)$ and used for statistical evaluation. Tests on non-linearity, normal distribution, homoscedasticity and influential outliers are done using residual vs. fitted, normal-Q-Q-, scale-location and residuals vs. leverage plots. Further, the relation between the variables is tested on mediation effects. Monte Carlo Analysis [16] is used to test whether indirect effects can be found. As mediation analysis has the challenge to generate high coverage and high power [27], Sobel-Test and Bootstrapping [13] are carried out additionally.

\section{Results}

Results show significant $(\mathrm{p} ; .01)$ effects for all assumed relations between situation familiarity, objective traffic complexity, subjective complexity and the time to action decision (Figs. 4 and 5). The two independent variables situation familiarity and objective traffic complexity do both influence the subjective complexity significantly. They also have a direct influence on the time to action decision. The time to make an action decision is additionally significantly influenced by the subjective complexity. Fig. 5 shows high deviations throughout the data. However, concerning subjective complexity this is not surprising as variations in subjective perceptions are expected. Variances in the time until an action decision is made base mostly on different monitoring behavior and hedging strategies. Some participants make the action decision before starting the hedging strategies, others make sure the maneuver is executable before taking the action decision. These deviations will be examined more closely based on eye-tracking measurements that have been collected additionally, but are not part of this paper. Here, the detailed relations between the described constructs are analyzed closely.

\subsection{The relation between Situation Familiarity and the Subjective Complexity (H1.1)}

Regression analysis between situation familiarity and the subjective complexity show that with a rise in familiarity with the situation, the subjective complexity is reduced significantly $(\beta=-0.83, p<.001$; Fig. 5). Situation familiarity explains $5 \%$ of variance $\left(R^{2}=.05, t(358)=\right.$ $-4.34, p<.001)$. As the overall impact of familiarity on subjective complexity is highly significant, the impact of each sub-scale on the total effect is examined. Sub-scales should not be analyzed separately, but can give a better understanding of how subjective complexity is influenced by situation familiarity. Taking a closer look at the separate characteristics of subjective complexity (Table 1), especially the subjective perception of performance and frustration is influenced by the familiarity with the situation. Participants have the feeling to perform better and are less frustrated when they are more familiar with a situation. Also mental and temporal demand perception decreases with a rise in familiarity as well as the subjective effort that is needed for the maneuver. Only the physical demand (e.g. steering, using gas- and brake pedals) is subjectively not reduced with a rise in situation familiarity.

\subsection{The relation between Situation Familiarity and the Time to Action Decision (H1.2)}

The familiarity with the situation does also directly influence the time until participants make their action decision. Overall, decision-times range from $\min =2.57 \mathrm{~s}$ to $\max =24.77 \mathrm{~s}$ with a mean of $M=6.74 \mathrm{~s}$, a Median of $2.25 \mathrm{~s}$ and a standard deviation of $S D=3.99$. With a rise in the familiarity with the situation due to repeated exposure of the scenarios, participants become significantly faster in their action decision (Table 2). This can also be shown in a regression analysis (Fig. 5). It shows a negative slope of $\beta=-1.13(p<.001)$ for the regression. The situation familiarity can explain 5\% of variance for the time to make an action decision $\left(R^{2}=.05, t(332)=-4.26, p<.001\right)$. 
Fig. 4 Significance and $\beta$ coefficients of the hypothesized relations between Situation Familiarity, Objective Traffic Complexity, Subjective Complexity and the Time to make an Action Decision (significance codes: 0 ' $* * *$, $0.001{ }^{\prime} * *, 0.01{ }^{\prime}, 0.05, .0 .1$, ' 1 ; source: own figure)
Fig. 5 Box-Whisker Plots display data distributions including results of regression analysis (red line) and significance of the regression results (significance codes: 0 '***' $0.0011^{*} *{ }^{\prime}, 0.01{ }^{\prime}{ }^{\prime}, 0.05$ ', $0.1,{ }^{\prime}, 1$; source: own figure)
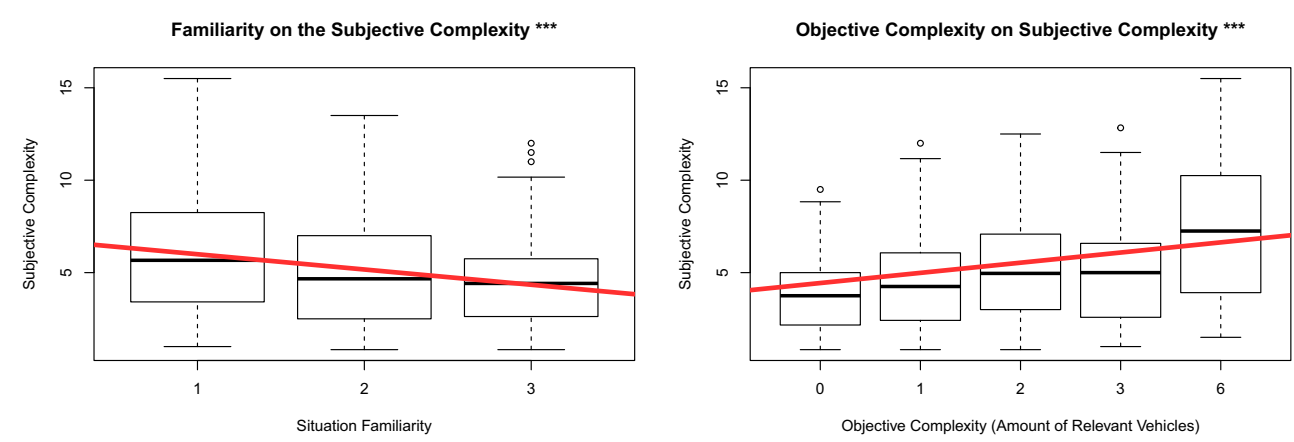

Familiarity on the Time to Action Decision ***
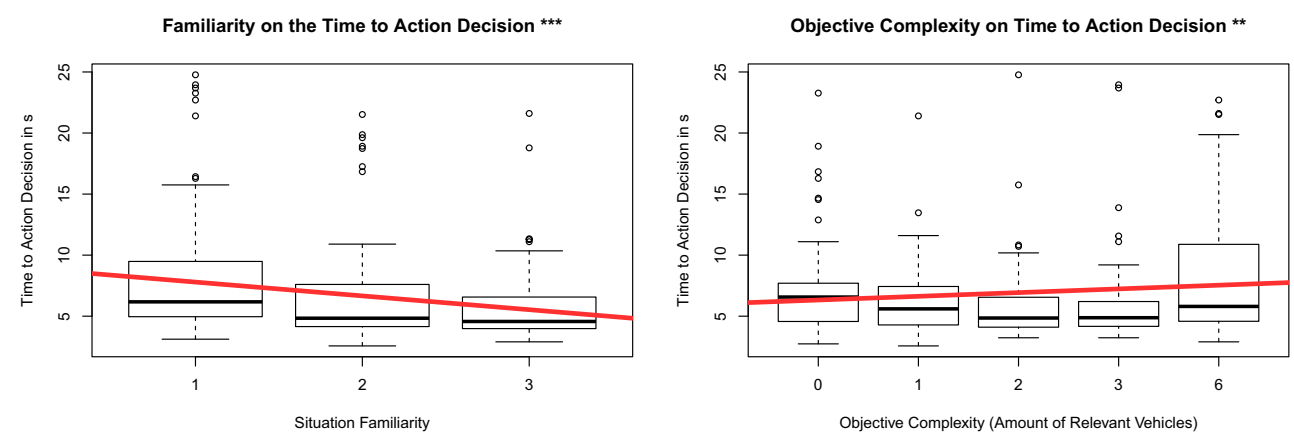

Situation

Familiarity

Objective Traffic

Complexity
Pseudo-

Dependent

Variable

Dependent Variable

Subjective

(Perceived)

Complexity

Time to Action Decision
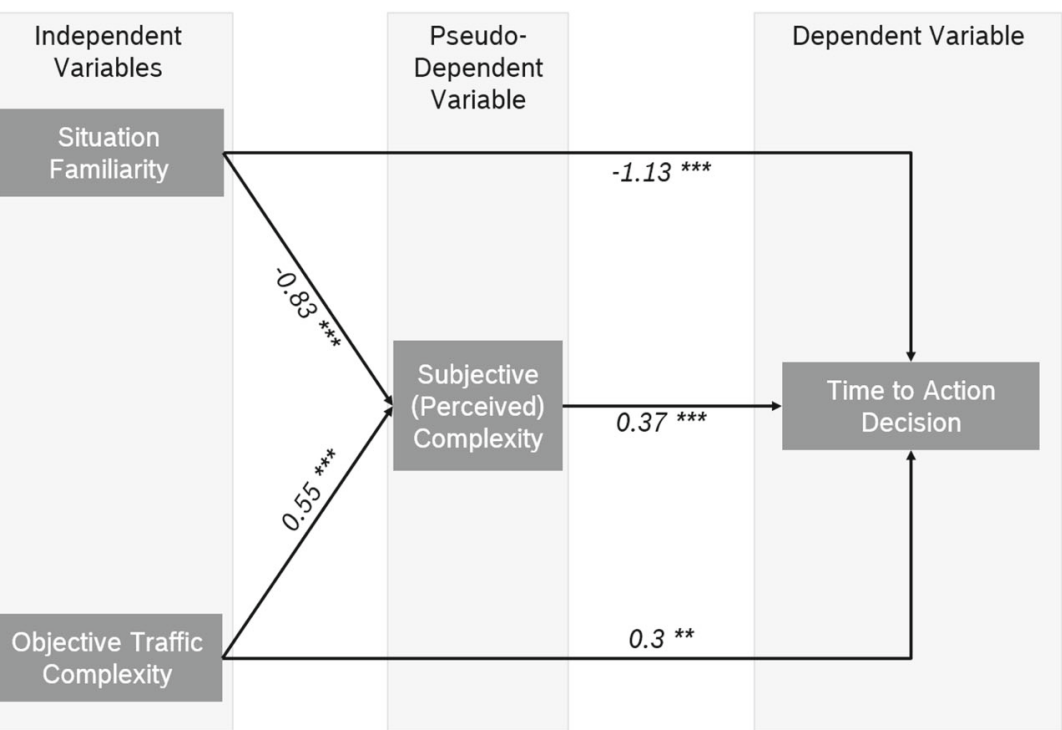

Table 1 Results of linear regression analysis between the situation familiarity on the separate characteristics of subjective complexity (significance codes: 0 '***' $0.001^{\prime} * * *^{\prime} 0.01^{\prime} *{ }^{\prime} 0.05^{\prime},{ }^{\prime} 0.1{ }^{\prime}, 1$; source: own table)

Subjective complexity

\begin{tabular}{llll} 
& $\beta$ & $t(358)$ & $R^{2}$ \\
\hline Total & -0.83 & -4.34 & $0.05 * * *$ \\
Mental Demand & -0.85 & -3.23, & $0.03 * *$ \\
Temporal Demand & -0.85 & -3.04, & $0.02 * *$ \\
Physical Demand & -0.28 & -1.7, & 0.01. \\
Performance & -1.08 & -3.85, & $0.04 * * *$ \\
Effort & -0.7 & -2.86, & $0.02 * *$ \\
Frustration & -1.21 & -5.41, & $0.07 * * *$ \\
\hline
\end{tabular}


Table 2 Action Decision Time statistics of participants in different conditions of Situation Familiarity

\begin{tabular}{|c|c|c|c|c|c|}
\hline \multirow[t]{2}{*}{ Situation Familiarity } & \multicolumn{5}{|c|}{ Time to action decision } \\
\hline & Min & Median & Mean & Max & SD \\
\hline 1 & $3.117 \mathrm{~s}$ & $6.176 \mathrm{~s}$ & $7.907 \mathrm{~s}$ & $24.768 \mathrm{~s}$ & 4.6 \\
\hline 2 & $2.566 \mathrm{~s}$ & $4.834 \mathrm{~s}$ & $6.425 \mathrm{~s}$ & $21.51 \mathrm{~s}$ & 3.79 \\
\hline 3 & $2.900 \mathrm{~s}$ & $4.567 \mathrm{~s}$ & $5.675 \mathrm{~s}$ & $21.602 \mathrm{~s}$ & 2.96 \\
\hline
\end{tabular}

Depiction of minimum (Min), median, mean, maximum (Max) values and the standard deviation (SD; source: own table)

\subsection{Subjective Complexity as Mediator between the influence of Situation Familiarity on the Time until an Action Decision is made (H1.3)}

Further it is hypothesized, that the impact of situation familiarity on time to action decision can be explained by the subjective complexity. It can be shown, that the subjective complexity has a significant influence on the time to make an action decision $\left(\beta=0.37, R^{2}=\right.$ $.08, t(332)=5.46, p<.001)$ and is thus a possible mediator between situation familiarity and the time to action decision (Fig. 6).

This means, that the direct influence disappears when adding the variable subjective complexity to the regression. Monte-Carlo Analysis does not show a significant effect of mediation, but as mentioned above (2.6) Sobel-Test and Bootstrapping are additionally carried out. Both show significant results for a mediation effect. The Sobel-Test shows a significant effect on a 5\% level $(t>1.96) .24 \%$ of the total effect can be traced back to the mediation effect. The same significant effect shows the Bootstrapping method with a mediation effect of -0.27 . Due to these results, the hypothesis, that subjective complexity mediates the influence of situation familiarity on time to action decision is accepted.

\subsection{The relation between Objective Complexity and Subjective Complexity (H2.1)}

Further, the impact of the objective complexity on the subjective complexity is tested. In a first step, high vs. low conditions are compared using a Mann-Whitney-U Test. The result indicates a significant difference ( $\mathrm{p}_{i} .05$ ) between the two groups (high and low complexity). To examine objective complexity closer, the amount of relevant vehicles in the traffic environment is manipulated in this study. Hence, an evaluation of the amount of relevant vehicles in the environment is carried out. Regression analysis is conducted to evaluate the slope of the relation between objective complexity, in terms of relevant vehicles in the traffic environment and subjective complexity. The amount of relevant vehicles for each scenario is described in Section 2.4.2. Results of the regression show, that with a rise in the amount of relevant vehicles in the traffic environment, the subjective complexity rises (Fig. 5). This influence is statistically highly significant and has a slope of $\beta=0.55$ $(p<.001)$. Twelve percent of variance in the subjective complexity can be explained by the amount of relevant vehicles in the traffic environment $\left(R^{2}=.12, t(358)=\right.$ $6.92, p<.001$; Table 3). Looking closer at the sub-scales (that should not be analyzed separately but give a good
Fig. 6 The mediation effect of Subjective Complexity between Situation Familiarity and Time to Action Decision (significance codes: $0{ }^{\prime} * *, 0.001, * *, 0.01$ '*' 0.05, ', 0.1 ', 1 ; source: own figure)

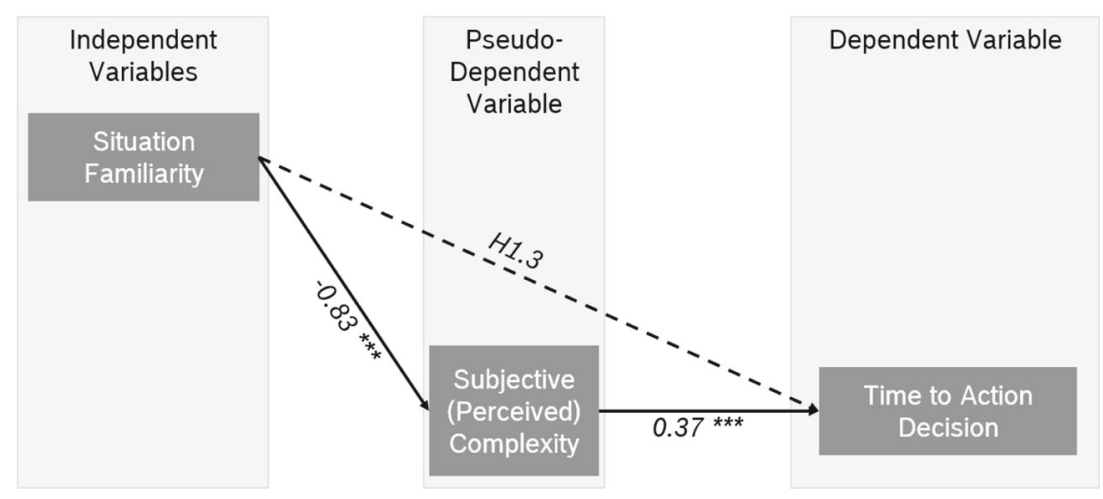


Table 3 Results of linear regression analysis between the objective traffic complexity on the separate characteristics of subjective complexity (significance codes: $0{ }^{\prime} * * *, 0.0011^{\prime} * *, 0.01{ }^{\prime} *{ }^{\prime}, 0.05$ '.' $0.1{ }^{\prime},{ }^{\prime} 1$; source: own table).

\begin{tabular}{llll}
\hline Subjective Complexity & Objective traffic complexity & & $t(358)$ \\
& $\beta$ & 6.92 & $R^{2}$ \\
\hline Total & 0.55 & 7.27 & $0.12 * * *$ \\
Mental Demand & 0.79 & 7.72 & $0.13 * * *$ \\
Temporal Demand & 0.88 & 3.92 & $0.14 * * *$ \\
Physical Demand & 0.27 & 2.94 & $0.04 * * *$ \\
Performance & 0.36 & 5.48 & $0.02 * *$ \\
Effort & 0.56 & 4.59 & $0.07 * * *$ \\
Frustration & 0.45 & $0.05 * * *$ \\
\hline
\end{tabular}

understanding of how objective complexity influences the subjective complexity), the following effects are found. Effort, frustration, mental-, temporal- and physical demand significantly increase with a rise of the amount of relevant surrounding vehicles, while the subjective performance significantly decreases (Table 3).

\subsection{The relation between Objective Complexity and the Time to Action Decision (H2.2)}

For statistical evaluation the amount of relevant vehicles in the environment is directly used here. Regression analysis between objective complexity and the time to make an action decision shows significant results. In Section 3.2 the overall statistics for the time to action decision are described $(M=6.74, S D=3.99)$. Table 4 shows the statistical results for the effect of each amount of relevant vehicles in the traffic environment on the time (in seconds) until an action decision is made. Although huge differences cannot be found, the effect that the amount of relevant vehicles in the environment has on the time to action decision is significant on a .01 level $\left(\beta=0.3, R^{2}=.02, t(332)=52.65, p<\right.$ $.01)$. Thus, with a rise in relevant vehicles, also the time until an action decision is made rises significantly (Fig. 5). Two percent of the variance in time to action decision can be explained by the amount of relevant vehicles in the environment.

\subsection{Subjective Complexity as Mediator between the influence of Objective Complexity on the Time until an Action Decision is made (H2.3)}

For the objective traffic complexity it is tested whether the subjective complexity has a mediating effect between objective complexity and time to action decision. The objective complexity significantly influences both, subjective complexity and time to action decision (Sections 3.4 and 3.5). Also, subjective complexity has a significant impact on time to action decision (Section 3.3). Based on this, it is assumed that subjective complexity is a mediator variable between objective complexity and the time to action decision (Fig. 7). As in Section 3.3, Monte-Carlo Analysis, Sobel-Test and Bootstrapping are executed to test whether a mediation effect exists. All three procedures show significant effects. Sobel-Testing shows, that $63 \%$ of the total effect can be traced back to the mediation effect and the mediation effect is 0.19 for Sobel and Bootstrapping. These results show, that subjective complexity mediates the influence between objective complexity and time to action decision.

Table 4 Action Decision Time statistics of participants in different conditions of objective complexity that is quantified as the amount of relevant vehicles in the traffic environment

\begin{tabular}{lllll}
\hline Relevant Vehicles & \multicolumn{2}{l}{ Time to action decision } & & \\
& Min & Median & Mean & Max \\
\hline 0 & $2.734 \mathrm{~s}$ & $6.567 \mathrm{~s}$ & $7.422 \mathrm{~s}$ & $23.269 \mathrm{~s}$ \\
1 & $2.566 \mathrm{~s}$ & $5.601 \mathrm{~s}$ & $6.476 \mathrm{~s}$ & $21.401 \mathrm{~s}$ \\
2 & $3.233 \mathrm{~s}$ & $4.850 \mathrm{~s}$ & $5.823 \mathrm{~s}$ & $24.768 \mathrm{~s}$ \\
3 & $3.233 \mathrm{~s}$ & $4.875 \mathrm{~s}$ & $6.139 \mathrm{~s}$ & $23.952 \mathrm{~s}$ \\
6 & $2.900 \mathrm{~s}$ & $5.800 \mathrm{~s}$ & $8.733 \mathrm{~s}$ & $22.701 \mathrm{~s}$ \\
\hline
\end{tabular}

Depiction of minimum (Min), median, mean, maximum (Max) values and the standard deviation (SD; source: own table) 
Fig. 7 The mediation effect of Subjective Complexity between the amount of relevant vehicles in the traffic environment and Time to Action Decision (significance codes: 0 ' $* * *$, $0.0011^{\prime} *{ }^{\prime}, 0.01{ }^{\prime}{ }^{\prime}, 0.05,5^{\prime}, 0.1$ '

' 1 ; source: own figure)

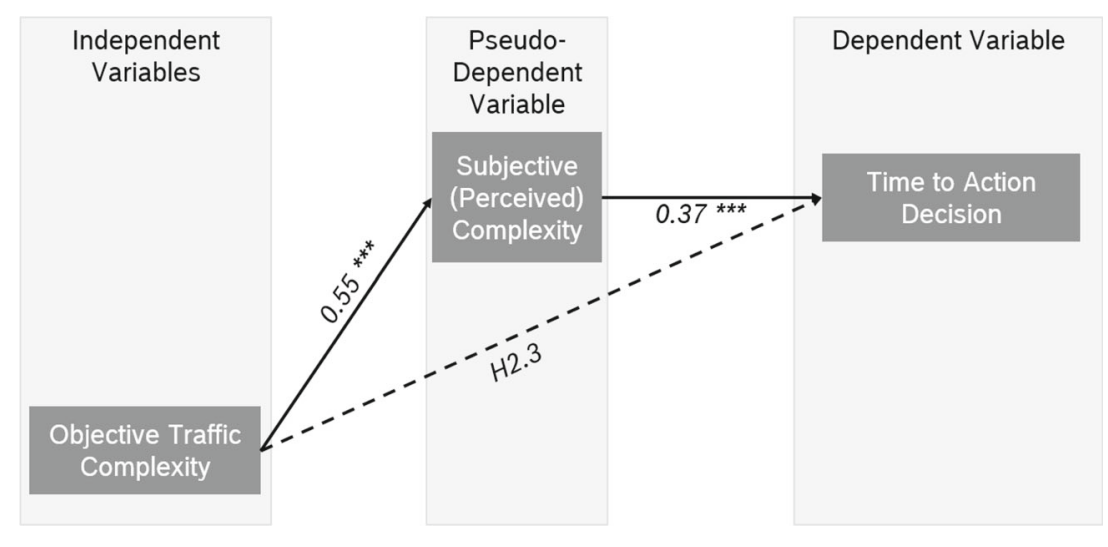

\section{Discussion}

The results show, that situation familiarity as well as the amount of relevant vehicles in the traffic environment have a significant impact on how demanding and complex a situation is perceived. The amount of variance that can be explained is 5\% for situation familiarity and $12 \%$ for objective complexity. Similarly, both variables have an influence on the time until an action decision is made. Again, situation familiarity can explain 5\% of variance, objective complexity $2 \%$ of variance. With highly significant results these two variables are influencing parameters for both, subjective complexity and time to action decision. Still, a lot of variance cannot be explained by the described variables and differences in decision times are marginal. Further parameters, that have an influence on situation familiarity and/or time to action decision are not yet investigated. In addition, the objective complexity did only focus on the amount of relevant vehicles in the traffic environment. Further objects in the surrounding traffic (e.g. traffic signs, head-up display, notifications in the interface) but also in the surrounding environment (e.g. animals at the roadside, weather conditions) might influence the subjective complexity as well. Individual aspects like selective attention, vigilance, primary goal etc. should be considered. Still, not all possible aspects that might influence the subjective complexity are possible to capture at once. The mediation effects, that have been found in this paper point out a solution that bypasses the necessity of capturing all of the possible influencing factors. As subjective complexity is a mediator variable for both, situation familiarity and objective complexity on the time to action decision, information about the perceived complexity of an individual can already serve as an indicator for action decision times. This finding is highly important, as it shows that measuring subjective complexity can be a solution for elaborating times until an action decision is made, predict those and adapt a cognitive assistance system accordingly. The time of making an action decision can be assumed to represent the time, when the situation awareness is fully built up. To support the driver in take-over situations, supplementary features such as gaze guidance or increased decelerations are necessary [4]. By knowing how the subjective complexity influences the process of building up situation awareness, cognitive assistance systems can be developed with respect to the individual perception of complexity in certain situations. Thus, a cognitive assistant could support an overstrained driver by reducing the speed, handing over the driving task gradually and giving maneuver indications. Braking, steering, lane keeping or navigation tasks can successively be adapted based on the individual drivers current state. A detailed representation of the environment including every possible distraction is thus not necessary. Especially in driving, different individuals perceive and respond to cues in different ways which are dependent on their current goal, their mood, vigilance etc.. By measuring subjective complexity of the individual, the prediction of Action Decision Times is possible. These predictions can extrapolate to the completion of building up situation awareness. In this study, the NASA-TLX is used to measure subjective complexity. In laboratory conditions this is useful to gather detailed information about the subjective complexity that a driver perceives. Still, in reality, other measurement methods for subjective complexity are necessary. A conceivable solution would be physiological or eye tracking data to measure how complex a situation is perceived. This field is still developing and more research is necessary here. In future research it is relevant to investigate the mediation effect further, replicate it and focus on useful methods to measure the subjective complexity fast and easily.

\section{Conclusion}

Results of this study provide a first understanding of the role that different complexity aspects play during the takeover in highly automated driving. It can be shown that subjective 
complexity, a highly individual variable, is very important to consider when developing driver assistance systems. The here presented mediation provides a first indication of an easy solution to individualize cognitive assistance systems without the need of capturing complex driver data. If a measurement of subjective complexity of the individual is possible, the adaption of assistance systems is already possible. Future studies should focus on solid methods to measure subjective complexity. Thus, individually adaptive driver assistance systems can be developed that propose a solution to induce smooth and safe transition to manual driving.

Acknowledgements I wish to acknowledge the help provided by my supervisors Kathrin Zeeb and Michael Schulz at Robert Bosch GmbH, my department at TU Berlin for support and my second supervisor Prof. Dr. Klaus Bengler from the TU Munich.

Author Contributions Conceptualization: Marlene Scharfe; Methodology: Marlene Scharfe; Formal analysis and investigation: Marlene Scharfe; Writing - original draft preparation: Marlene Scharfe; Writing - review and editing: Marlene Scharfe and Nele Russwinkel; Resources: Marlene Scharfe; Supervision: Nele Russwinkel.

Funding Open Access funding enabled and organized by Projekt DEAL. This work is part of the public promoted project PAKoS in which the Robert Bosch GmbH participated.

\section{Declarations}

Informed Consent The authors declare that they have no conflict of interest. The study was approved by the ethics committee of the TU Berlin.

Open Access This article is licensed under a Creative Commons Attribution 4.0 International License, which permits use, sharing, adaptation, distribution and reproduction in any medium or format, as long as you give appropriate credit to the original author(s) and the source, provide a link to the Creative Commons licence, and indicate if changes were made. The images or other third party material in this article are included in the article's Creative Commons licence, unless indicated otherwise in a credit line to the material. If material is not included in the article's Creative Commons licence and your intended use is not permitted by statutory regulation or exceeds the permitted use, you will need to obtain permission directly from the copyright holder. To view a copy of this licence, visit http://creativecommons. org/licenses/by/4.0/.

\section{References}

1. Addanki, S.C., et al.: Analysis of Traffic Related Factors and Vehicle Environment in Monitoring Driver's Driveability. In: International Journal of Intelligent Transportation Systems Research, pp. 1-11 (2019)

2. Ampore, E., et al.: Cognitive systems in intelligent vehicles - a new frontier for autonomous driving. In: Proceedings of the 11th International Conference on Informatics in Control, Automation and Robotics - ICINCO 2014. https://doi.org/10.5220/0005160808170822 (2014)
3. Baumann, M., Krems, JF.: Situation awareness and driving: A cognitive model. In: Modelling driver behaviour in automotive environments, pp. 253-265 (2007)

4. Braunagel, C., Rosenstiel, W., Kasneci, E.: Ready for Take-Over? A New Driver Assistance System for an Automated Classification of Driver Take-Over Readiness. In: IEEE Intell. Transp. Syst. Mag. 9.4, pp. 10-22. https://doi.org/10.1109/MITS.2017. 2743165 (2017)

5. Damböck, D., Bengler, K.: Übernahmezeiten Beim Hochautomatisierten Fahren. In: 5. Tagung Fahrerassistenz (2012)

6. Endsley, M.R.: From here to autonomy: lessons learned from human-automation research. Hum. Fact. 59.1, 5-27 (2017)

7. Endsley, M.R.: Toward a theory of situation awareness in dynamic systems. Hum. Fact. 37.1, 32-64 (1995)

8. Estes, S. et al.: Digital copilot: Cognitive assistance for pilots. In: Proceedings of 2016 AAAI Fall Symposium Series (2016)

9. Gross, U.: Bestimmung von Schwierigkeitsgraden in einer zu entwickelnden Versuchsumgebung. In: Diplomarbeit - Humbold UniversitäT Zu Berlin, Germany (2004)

10. Haerem, T., Rau, D.: The Influence of Degree of Expertise and Objective Task Complexity on Perceived Task Complexity and Performance. J. Appl. Psychol. 92.5, 1320 (2007)

11. Hart, S.G.: NASA Task Load Index (TLX). In: From Nasa - Ames Research Center. vol. 1 (1986)

12. Kasuga, N., et al.: Design of an HMI System Promoting Smooth and Safe Transition to Manual from Level 3 Automated Driving. Int. J. Intell. Transp. Syst. Res. 18.1, pp. 1-12 (2020)

13. Koopman, J., Howe, M., Hollenbeck, J.R.: Pulling the Sobel test up by its bootstraps. In: More statistical and methodological myths and urban legends, pp. 224-244 (2014)

14. Larsson, A.F.L., Kircher, K., Hultgren. JA: Learning from experience: Familiarity with ACC and responding to a cut-in situation in automated driving. In: Transp. Res. Part F: Traffic Psychol. Behav. 27, 229-237 (2014)

15. Louw, T., Merat, N., A.Jamson.: Engaging with highly automated Driving: To be or not to be in the loop? In: Proceedings of 8th International Driving Symposium on Human Factors in Driver Assessment, Training and Vehicle Design. Salt Lake City. https://doi.org/10.13140/RG.2.1.2788.9760 (2015)

16. MacKinnon, D.P., Lockwood, C.M., Williams, J.: Confidence limits for the indirect effect: Distribution of the product and resampling methods. Multivar. Behav. Res. 39.1, 99-128 (2004)

17. Maynard, D.C., Hakel, M.D.: Effects of objective and subjective task complexity on performance. Hum. Perform. 10.4, 303-330 (1997)

18. McKnight, P.E., Najab, J.: Mann-Whitney U Test. In: The Corsini encyclopedia of psychology, pp. 1-1 (2010)

19. Paxion, J., Galy, E., Berthelon. C: Overload depending on driving experience and situation complexity: which strategies faced with a pedestrian crossing? Appl. Ergon. 51, 343-349 (2015)

20. Plavsic, M.: Analysis and Modeling of Driver Behavior for Assistance Systems at Road Intersections. In: Ph.D. Thesis Technische UniversitäT München (2010)

21. Radlmayr, J., et al.: How Traffic Situations and Non-Driving Related Tasks Affect the Take-Over Quality in Highly Automated Driving. Proc. Hum. Fact. Ergon. Soc. Ann. Meet. 58.1, 20632067. https://doi.org/10.1177/ 1541931214581434 (2014)

22. Razali, N.M., Wah, Y.B., et al.: Power comparisons of shapirowilk, kolmogorovsmirnov, lilliefors and anderson-darling tests. J. Stat. Model. Anal. 2.1, 21-33 (2011)

23. SAE-International: Surface Vehicle Recommended Practice. Taxonomy and Definitions for Terms Related to On-Road Motor Vehicle Automated Driving Systems. In: SAE International (2018)

24. Scharfe, MS.L., Russwinkel, N.: A Cognitive Model for Understanding the Takeover in Highly Automated Driving Depending 
on the Objective Complexity of Non-Driving Related Tasks and the Traffic Environment. In: Proceedings of the 41th Annual Cognitive Science Society Meeting, pp. 2734-2740. https://cognitivesciencesociety.org/wpcontent/uploads/2019/07/ cogsci19_proceedings-8July2019-compressed.pdf (2019)

25. Schlindwein, S.L., Ison, R.: Human knowing and perceived complexity: implications for systems practice. Emerg. Complex. Organ. 6.3, 27-32 (2004)

26. Suzuki, K., et al.: Performance evaluation of a crossing-assistance system for visually disabled persons at intersections considering actual road structure. Int. J. Intell. Transp. Syst. Res. 8.1, 26-35 (2010)

27. Tofighi, D., MacKinnon, D.P.: Monte Carlo confidence intervals for complex functions of indirect effects. Struct Equ. Model. Multidiscip. J. 23.2, 194-205 (2016)

28. WIVW-GmbH. Driving Simulation and SILAB (2014). https:// wivw.de/en/silab (visited on 07/09/2019)

29. Yang, B., et al.: Evaluation of Driver Assistance System Presenting Information of Other Vehicles through Peripheral Vision at Unsignalized Intersections. In: International Journal of Intelligent Transportation Systems Research, pp. 1-10 (2020)

30. Yang, B., et al.: Evaluations of Different Human Machine Interfaces for Presenting Right-Turn Timing at Intersections. In: International Journal of Intelligent Transportation Systems Research, pp. 1-12 (2020)

Publisher's Note Springer Nature remains neutral with regard to jurisdictional claims in published maps and institutional affiliations.

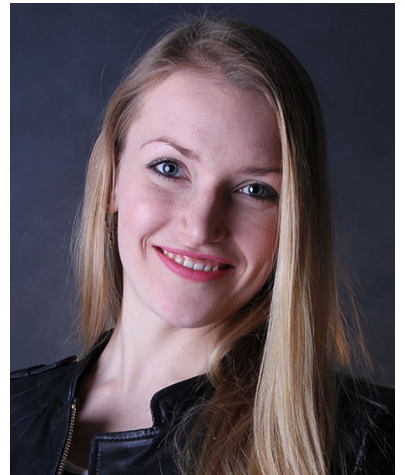

Marlene Susanne Lisa Scharfe-Scherf was a doctoral student at Technical University Berlin in cooperation with Robert Bosch $\mathrm{GmbH}$. Her research includes ACT-R cognitive modeling of the takeover process in highly automated driving, including the process to build up situation awareness. Using eye tracking and a driving simulator, she validated the cognitive model for the takeover in highly automated driving.

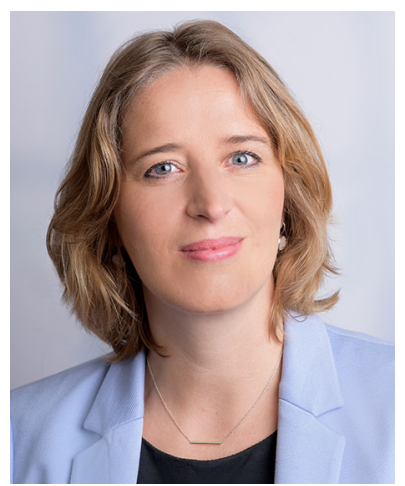

Nele Russwinkel studied Cognitive Science at the university of Osnabrück. Her master thesis about visual attention was a collaboration with the Humboldt University and the Charitè, Berlin. Nele Russwinkel started at the TU Berlin in 2005 as research assistant and graduated 2010 . Since 2013 she holds the chair of "cognitive modelling in dynamic human system interaction" at the institute of psychology and ergonomics at the TU Berlin. 